\title{
Pratique du séchage artisanal de fruits et légumes dans le sud du Cameroun
}

\author{
Marcel Edoun ${ }^{1,2 \star}$, Alexis KUITCHE ${ }^{1}$, Claude MAROUZÉ ${ }^{2}$, François GIROUX ${ }^{2}$, César KAPSEU ${ }^{1}$
}

\author{
${ }^{1}$ École Ntl. Sup. Sci. Agro-Ind., \\ BP 455, Ngaoundéré, \\ Cameroun, \\ edounmarcel@yahoo.fr \\ 2 CIRAD, Persyst, \\ UMR QUALISUD, TA 40/15, \\ 73 , rue J.F. Breton, \\ 34398 Montpellier, Cedex 5, \\ France
}

* Correspondance et tirés à part

Reçu 23 septembre 2009

Accepté 2 février 2010

Fruits, 2011, vol. 66, p. 25-36

(C) 2011 Cirad/EDP Sciences

All rights reserved

DOI: 10.1051/fruits/2010038

www.fruits-journal.org

RESUMEN ESPAÑOL, p. 36

\section{Practice of the craft of drying fruits and vegetables in southern Cameroon.}

Abstract - Introduction. In recent years, the drying of fruits and vegetables on a small scale has experienced a resurgence of interest in Cameroon, both for local sale and export. However, processors who engage in this activity are not satisfied with the dryers they use. Materials and methods. In this context, we conducted a semi-open survey of fifty drying units installed in the areas of Douala and Yaoundé (Cameroon) to identify better the challenges they face. Information obtained during this survey was coded to facilitate the IT operations. The final sample constituted only companies working on a small scale ( $70 \%$ of the initial sample), that is to say, treating less than $500 \mathrm{~kg}$ of fresh produce per drying cycle. Results. The analysis results showed that nearly $45 \%$ of units surveyed did not have a dryer and that $70 \%$ of the dryers in the units surveyed were equipped with gas dryers. Overall, nearly $92 \%$ of these dryers are natural convection dryers. The most well-known dryer model is the Atesta model, imported from Burkina Faso, variants of which are manufactured locally. It turns out that this model only partially meets the needs of users and local processors, who are increasingly abandoning the dryers. Conclusion. The inventory of fixtures of the activity of small-scale drying of fruits and vegetables in southern Cameroon revealed that, despite the different offers locally available, the need for drying devices that meet user expectations better is very important. This need translates into a willingness to acquire dryers with the capacity to treat over $80 \mathrm{~kg}$ of fresh produce during a 10-h drying cycle, and equipped with a ventilation and control system of drying parameters.

Cameroon / humid tropics / tropical fruits / vegetables / drying dehydration / forced air drying / dryers / handicrafts / small enterprises

Pratique du séchage artisanal de fruits et légumes dans le sud du Cameroun.

Résumé - Introduction. Depuis quelques années, le séchage des fruits et légumes à petite échelle connait un regain d'intérêt au Cameroun, que ce soit pour la vente locale ou pour celle d'exportation. Or, les transformateurs qui pratiquent cette activité ne sont pas satisfaits des séchoirs qu'ils utilisent. Matériel et méthodes. Dans ce contexte, nous avons réalisé une enquête de type semi ouvert auprès d'une cinquantaine d'unités de séchage installées dans les zones de Douala et de Yaoundé (Cameroun) afin de mieux identifier les difficultés qu'elles rencontrent. Les informations obtenues au cours de cette enquête ont été codifiées pour faciliter leur exploitation informatique. Nous n'avons conservé dans l'échantillon constitué que les entreprises travaillant à petite échelle (70\% de l'échantillon initial), c'est-à-dire traitant moins de $500 \mathrm{~kg}$ de produits frais par cycle de séchage. Résultats. L'analyse des résultats a montré que près de $45 \%$ des unités étudiées ne possédaient pas de séchoir et que 70 \% des séchoirs recensés dans les unités qui en étaient équipées étaient des séchoirs à gaz. Dans l'ensemble, près de $92 \%$ de ces séchoirs sont des séchoirs à convection naturelle. Le modèle de séchoir connu est le modèle Atesta importé du Burkina Faso dont des variantes sont fabriquées localement. Il s'avère que ce modèle ne répond que partiellement aux besoins des utilisateurs et que les transformateurs locaux abandonnent de plus en plus ces séchoirs. Conclusion. L'état de lieux des activités de séchage à petite échelle des fruits et légumes dans le sud du Cameroun a fait apparaître que, malgré les différentes offres disponibles localement, le besoin en dispositifs de séchage répondant mieux aux attentes des utilisateurs reste très important. Ce besoin se traduit par une volonté d'acquérir des séchoirs présentant la capacité de traiter plus de $80 \mathrm{~kg}$ de produits frais durant un cycle de séchage de $10 \mathrm{~h}$, et équipés d'un système de ventilation et de régulation des paramètres de séchage.

Cameroun / tropiques humides / fruits tropicaux / légume / séchage déshydratation / séchage par ventilation / séchoir / artisanat / petite entreprise 


\section{Introduction}

De multiples efforts ont été faits pour améliorer la productivité agricole au Cameroun [1, 2]. Malheureusement, de nombreuses pertes après-récoltes sont encore déplorées dans les zones de production ou de commercialisation de ces produits. En Afrique subsaharienne, ces pertes sont estimées à plus de $40 \%$ [3]. Pour faire face à ce problème, le séchage est une technique de conservation accessible à tous [4]. Dans ce contexte, le séchage solaire en particulier est une méthode dont la mise en œuvre est relativement facile [5]. Malheureusement, en zone tropicale humide, l'énergie solaire est insuffisante et son utilisation comme source principale d'énergie lors du séchage des fruits et légumes conduirait à accroître les pertes à cause d'un temps de séchage trop long. De ce fait, de nombreuses études ont été entreprises afin de proposer des types de séchoirs adaptés aux produits à sécher [6-10]. Malgré cela, la filière séchage des fruits et légumes n'a pas connu de forte expansion au cours des dix dernières années ; il en résulte même, par endroits, la fermeture d'unités de séchage existantes.

Pour tenter de trouver une solution durable à ce problème de développement, les organismes non gouvernementaux essayent d'organiser cette filière. À cette fin, ils ont entrepris de former des personnes à la création et à la bonne conduite d'unités de séchage à petite échelle pour le marché des produits biologiques. Ainsi, près de 300 opérateurs ont été formés à ces activités au cours de ces dernières années et cela principalement au Sud du Cameroun.

La région du sud du Cameroun est caractérisée par une très forte production agricole et un climat peu favorable au séchage [2], car l'hygrométrie de l'air y est relativement élevée (supérieure à $70 \%$ ) et la pluviométrie est forte tout au long de l'année (de $1700 \mathrm{~mm}$ à $4000 \mathrm{~mm}$ de pluie selon les endroits). Dans ce contexte, il s'avère particulièrement difficile de sécher des produits agricoles à haute teneur en eau dans des séchoirs à air. Le but de notre travail a donc été d'analyser les pratiques du séchage des produits en usage dans cette zone et de proposer des voies d'amélioration pour résoudre les problèmes rencontrés. Cette analyse a été effectuée à l'aide d'une enquête menée auprès d'unités de séchage à petite échelle traitant des fruits et des légumes dans le Sud du Cameroun entre mai 2007 et juin 2008.

\section{Matériel et méthodes}

\subsection{Collecte des informations \\ 2.1.1. Visite de terrain et déroulement de l'enquête}

Une première fiche d'enquête élaborée en préalable à notre analyse des pratiques de séchages au Sud du Cameroun nous a permis de tester le questionnaire auprès d'un nombre limité d'entreprises. Ces premières visites ont eu pour but de mettre en évidence les lacunes de cette première fichetest et de nouer des contacts avec des chefs d'unités cibles de notre étude. À l'issue de cette première série de visites, nous avons mis au point, puis adopté, le questionnaire final qui a été utilisé pour la suite de l'enquête (tableau I).

Les rendez-vous avec les chefs d'unités ont été pris au fur et à mesure du déroulement de l'enquête, à raison de deux à trois unités de séchage visitées quotidiennement. Lors de la première année, seules les unités implantées dans la zone de Douala ont été visitées. Durant la deuxième année, toutes les unités ont été visitées.

\subsubsection{Outils utilisés lors de l'enquête}

Un questionnaire de type semi-ouvert a été élaboré. Lors des visites d'information, un caméscope numérique a permis d'enregistrer les opérations de séchage in situ. L'air de séchage a été caractérisé par sa température et son hygrométrie (enregistreur Escort-Précision, Escort Data Loggers INC, USA) enregistrées chaque minute. Enfin, la vitesse de l'air de séchage a été mesurée par un anémomètre à fil chaud (Testo 405-V1, Testo NVISA, Allemagne).

Les réponses du type " oui / non " collectées sur les fiches d'enquêtes ont été codifiées par les modalités 0 et 1 : le code " 0 " a été donné pour une réponse négative et le code " 1 " pour une réponse positive. 
Tableau I.

Questionnaire utilisé auprès d'unités de séchage de fruits et légumes enquêtées dans le sud du Cameroun, afin de caractériser leur activité.

\section{Nature de la structure}

SARL / ONG / Association féminine / GIC / Autres

2. Niveau scolaire de l'opérateur

CEP / BEPC / BAC / DUT / Autres

3. Type de produits séchés

Fruits / Légumes / Tubercules / Feuilles / Viande / Poisson / Autre

Taille des produits ou morceaux (durée de séchage)

4. Période de forte activité : Novembre-Janvier / Mai- Septembre / Toute l'année / Autre Type de production : Batch / Continu

5. Qui achète vos produits secs ? Exportateur / Propriétaire de boutique local / Particulier

(a) Coût d'achat ou de revient du produit frais en FCFA

(b) Coût de revient du kg de produit séché en FCFA

6. Comment estimez vous que le produit est sec ?

Au touché / Nombre de jours mis dans le séchoir / Couleur du produit / Odeur

\section{Quel moyen avez-vous pour mesurer les paramètres de séchage ?}

Thermomètre / Hygromètre / Rien

8. Energie du séchoir utilisé

Séchoir électrique / Séchoir à gaz / Séchoir à charbon / Séchoir à bois / Séchoir solaire / Autre

9. Séchoir ventilé : Oui / Non

\section{Coût et date d'achat du séchoir}

11. Où avez-vous acheté votre séchoir?

Local (équipementier moderne ou artisan) / Importé

12. Comment avez-vous financé l'achat de votre séchoir?

Propre financement / Crédit communautaire / Offre non gouvernementale / Don

13. Qu'est-ce-qui a motivé votre choix pour ce séchoir et pas un autre ?

Prix / Capacité de séchage / Énergie utilisée / Système de financement / Adaptation au type de produit séché

14. Quel poste vous coûte cher au cours du processus de séchage ?

Energie / Main-d'œuvre / Matière première

15. Etes-vous satisfait de votre séchoir ? Très satisfait / Satisfait / Passable / Pas du tout

16. Avez-vous suivi une formation pour son utilisation ? Oui / non

17. Qui entretient et maintient votre séchoir?

Vous-même / Le fabricant ou vendeur / Un technicien local / un technicien venant d'une autre ville

18. Coût énergétique d'un $\mathbf{k g}$ de produit séché ou d'un kg de produit frais (ou idée de ce coût) Quelle est la consommation énergétique / batch?

Nombre de batch par semaine et durée d'un batch ?

19. Disponibilité de la source d'énergie ? Disponible / Rare / Manquante

20. Quelle opération effectuez-vous avant le séchage ? Épluchage / Pelage / Découpage

21. Que faites vous des déchets et épluchures des produits ? Jeter / Sécher et brûler / Vendre

\section{Problèmes rencontrés ?}

Détérioration des claies / Produit ne sèche pas bien / Délestage électrique / Pénurie du gaz / Procédé mal maitrisé / Paramètre non contrôlé / Emballage / Produit qui change de couleur / Séchoir non fiable / Facture énergétique / Pénurie de main-d'œuvre / Pénurie de matière première 


\section{Edoun et al.}

23. Vos besoins?

Capacité de séchage / Durée de séchage / Claie non sensible à la température / Séchoir ventilé / Utilisation des déchets et épluchures des produits pour la production de l'énergie

\section{Distance par rapport à vos principaux acheteurs $(\mathbf{k m})$ ?}

25. Quel est le montant maximum que vous pouvez dépenser pour l'achat d'un meilleur séchoir. Il est inutile de poser cette question sans donner un ordre de grandeur de capacité, de caractéristiques $<100000$ FCFA / 100.000 à 500.000 FCFA / > 500.000 à 1 MFCFA / > 1 MCFA

26. Classifier les critères de choix d'un séchoir

Prix / Capacité de séchage / Énergie utilisée / Type de produit séché / Complexité du fonctionnement / Facilité de maintenance / Polyvalence du séchoir / Coût de fonctionnement / Disponibilité locale

Figure 1.

Répartition de 50 unités de séchage de fruits et légumes étudiées dans le sud du Cameroun en fonction du type d'organisation dont elles font partie.

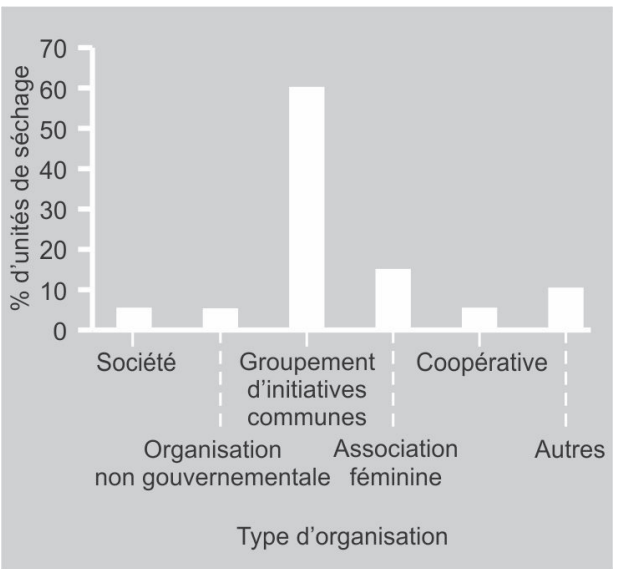

\section{2. Échantillonnage}

Les unités de séchage visitées ont été choisies dans la liste des structures faisant partie de la coopérative des sécheurs des produits Bio du Cameroun et parmi les entreprises Agro-alimentaires encadrées par le Centre pour l'Environnement et le Développement (CED) à Yaoundé (Cameroun).

Les unités de séchage identifiées sur ces listes ont ensuite été sélectionnées pour notre étude en fonction des deux critères :

(1) sur le type de produits soumis au séchage, en l'occurrence, pour nous, traitement des fruits et légumes,

(2) sur la localisation de la structure : dans notre cas, implantation en zone forestière humide à pluviométrie bimodale ou zone forestière humide à pluviométrie monomodale.

Ainsi cinquante unités respectant ces critères ont été visitées.

\section{Résultats et discussion}

\subsection{Structure des unités de séchage étudiées}

Sur l'ensemble des unités visitées qui pratiquent le séchage des fruits et des légumes au Cameroun, il s'est avéré que $60 \%$ d'entre elles appartenaient à des Groupements d'Initiatives Communes (GIC), $15 \%$ à des associations féminines, $5 \%$ à des Sociétés à Responsabilité Limitée (SARL), $5 \%$ également à des Organisations Non Gouvernementales (ONG) et $5 \%$ enfin à des coopératives ; les $10 \%$ restants étaient rattachés à d'autres structures (figure 1). Les GIC sont dirigés à $80 \%$ par des femmes.

La majorité (74\%) des unités visitées est installée dans les grandes métropoles (Douala, Yaoundé) et le reste, dans des agglomérations rurales situées en périphérie. La capacité journalière de séchage a été mesurée par la masse de produit frais que peut contenir un séchoir ou l'ensemble des séchoirs de l'unité par cycle de séchage de 24 heures. Sur cette base, il a été estimé que $60 \%$ des unités avaient une capacité de séchage inférieure à $50 \mathrm{~kg} \cdot \mathrm{cycle}^{-1}, 14 \%$ avaient une capacité de séchage comprise entre $(50$ et 100$) \mathrm{kg} \mathrm{cycl}^{-1}, \quad 10 \%$ se situaient dans la fourchette de (100 à 500) $\mathrm{kg} \cdot \mathrm{cycle}^{-1}$ et $16 \%$ disposaient d'une capacité supérieure à $500 \mathrm{~kg} \cdot \mathrm{cycle}^{-1}$ (figure 2).

La forte proportion des Groupes d'Intérêt Commun que nous avons constatée peut s'expliquer par le fait que les GIC au Cameroun sont exonérés d'impôts. La proportion élevée des femmes à la tête de ces GIC est 
une conséquence de la nouvelle politique gouvernementale au Cameroun qui encourage les actions visant l'épanouissement de la femme afin de lutter contre la pauvreté. Il apparaît que plus de 4/5 des unités étudiées avaient une capacité de séchage inférieure ou égale à $500 \mathrm{~kg}$ de produit frais par cycle de séchage de $24 \mathrm{~h}$. Pour ce critère, notre échantillon d'étude a été constitué de 42 unités de séchage dites " unités ou entreprises de séchage à petite échelle dans le sud Cameroun ".

\subsection{Les équipements de séchage existants}

À partir de l'enquête effectuée, il est apparu que près de $45 \%$ des unités de séchage dites à petite échelle ne possédaient pas de séchoir (figure 3) et devaient donc recourir à la location journalière d'un séchoir lors de leur d'activité. Par ailleurs, près de $70 \%$ des séchoirs répertoriés utilisent le gaz comme source d'énergie, les autres utilisant soit le soleil, soit le bois, soit le charbon de bois (figure 3). Nous n'avons pas observé d'unités utilisant un séchoir électrique. En outre, près de $92 \%$ des séchoirs se sont révélés être des séchoirs à convection naturelle, donc sans utilisation de ventilateur électrique et $60 \%$ des séchoirs que nous avons observés sont fabriqués par des artisans locaux. Cependant, le coût de l'énergie et les caractéristiques des produits sont très peu pris en compte par ces artisans-fabricants. Après quelques mois d'utilisation, les séchoirs proposés dans ce contexte sont abandonnés (figure 4) pour de multiples raisons : d'une part, ils ne permettent pas aux transformatrices d'obtenir des produits de qualité acceptable pour une éventuelle commercialisation ; d'autre part, la consommation énergétique élevée rend leur exploitation non rentable dans le contexte du sud du Cameroun. La faiblesse du séchoir à combustion réside dans son dispositif de chauffage de l'air et dans sa cellule de séchage. Pour ce type de séchoir, un opérateur est mobilisé pendant les heures de nuit pour assurer l'alimentation en combustible du foyer. Dans le contexte du sud du Cameroun, ce combustible peut être constitué soit des résidus de bois (copeaux, sciures de

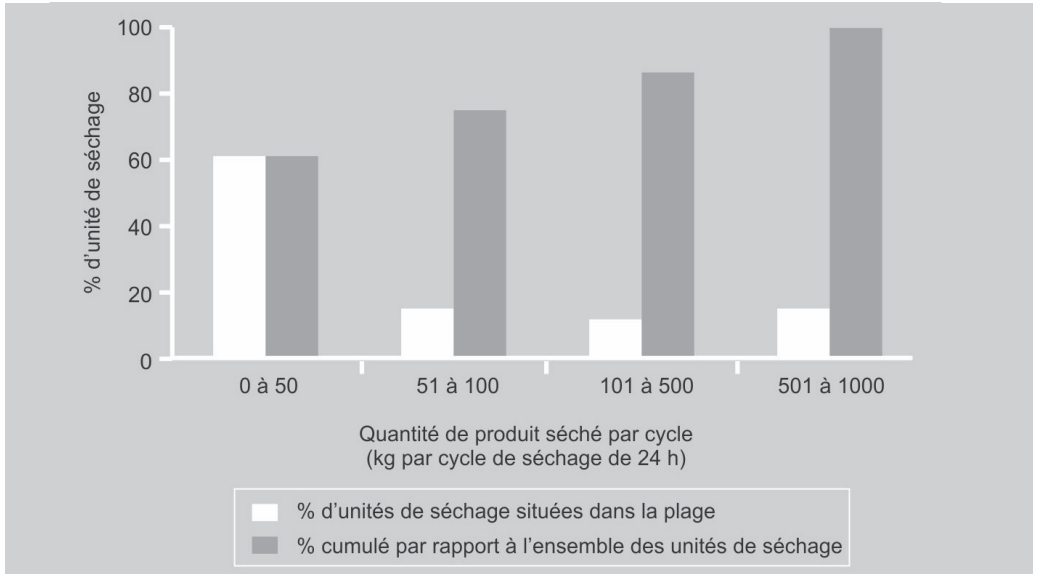

Figure 2.

Répartition de 50 unités de séchage de fruits et légumes étudiées dans le sud du Cameroun en fonction de la quantité de produit frais traitée par cycle de $24 \mathrm{~h}$.

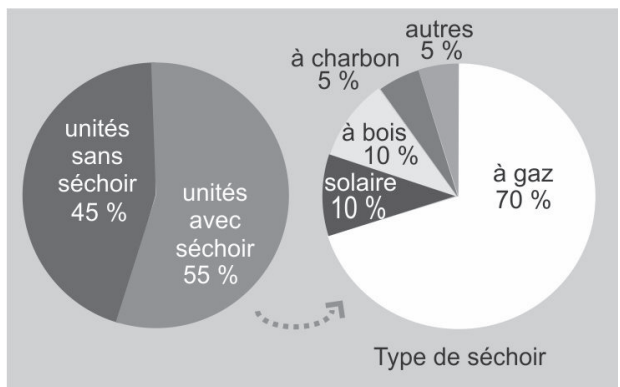

Figure 3.

Répartition de 42 unités de séchage de fruits et légumes étudiées dans le sud du Cameroun en fonction de l'équipement dont elles disposent.

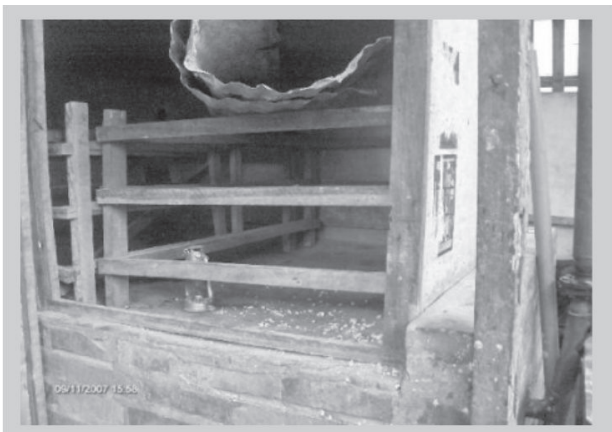

Figure 4.

Séchoirs de fabrication artisanale abandonnés par les transformateurs. La configuration de la cellule de séchage du séchoir à gaz entraine une surconsommation énergétique (13 kg de gaz pour l'obtention de $4 \mathrm{~kg}$ d'ananas séché). 
Tableau II.

Critère principal de choix pour l'acquisition d'un séchoir par les unités de séchage des fruits et légumes enquêtées dans le sud du Cameroun.

$\begin{array}{cc}\text { Critères de choix de séchoir } & \text { Proportion d'unités } \\ \text { ayant ce critère comme 1er critère de choix }\end{array}$

ayant ce critère comme
$(\%)$

Connaissance du séchoir

Énergie utilisée par le séchoir

Prix d'achat du séchoir

Capacité du séchoir

Système de financement

lors de l'achat du séchoir
42

34

8

8

8 bois, etc.), soit des épluchures des fruits traités dans l'unité. Pour le séchoir à gaz, après quelques temps d'utilisation, les claies ont tendance à se déformer et se déchirer, les battants à se détacher de l'ossature principale et la cellule de séchage apparaît donc non isolée.

\subsection{Critères de choix pour l'achat d'un séchoir}

À la question "qu'est-ce qui a motivé le choix de votre séchoir?", $42 \%$ des chefs d'unité ont indiqué s'être basés sur le fait d'avoir déjà vu le séchoir chez un autre transformateur (centre de formation, une amie, etc.), $34 \%$ ont dit avoir privilégié la source d'énergie utilisée par le séchoir; seuls $8 \%$ se sont intéressés en premier lieu au prix d'achat ou au système de financement du séchoir (tableau II). Contrairement à ce qu'on aurait pu attendre, la disponibilité du séchoir sur le marché et la capacité personnelle de financement se sont révélées ne pas constituer des critères essentiels de choix.

\subsection{Financement et achat des séchoirs}

Pour l'achat de leur séchoir, $60 \%$ des chefs d'unité de séchage ont recours aux crédits institutionnels [Fonds National de l'Emploi (FNE), micro finance] ; $30 \%$ au crédit communautaire (cotisation des membres du GIC ou de l'association) ; seuls $10 \%$ parviennent à financer personnellement l'achat de leur séchoir. Les crédits institutionnels représentent $2 / 3$ du montant global du coût d'achat du séchoir alors que les crédits communautaires peuvent couvrir jusqu'à $100 \%$ de ce coût.

Ces chiffres montrent que les centres de formation et les établissements de crédit ont une influence et peuvent influer sur le choix d'un séchoir. Les structures de micro-finance et/ou ONG proposent aux transformateurs les séchoirs qu'ils veulent vulgariser ou dont ils veulent faire la promotion. Bien que ces séchoirs ne répondent pas toujours aux besoins des transformateurs, ils sont parfois obligés d'accepter l'offre. C'est le cas du FNE qui propose aux jeunes créateurs d'entreprises des séchoirs à gaz à convection naturelle d'une capacité voisine de $80 \mathrm{~kg}$ de produit frais par cycle de $24 \mathrm{~h}$. Les responsables d'unité ayant effectué un stage dans un centre de formation ont tendance à choisir un séchoir identique à celui utilisé dans le centre de formation, d'où la proportion élevée des séchoirs à gaz à convection naturelle constatée dans les unités de séchage des produits biologiques à petite échelle implantées dans le sud du Cameroun.

\subsection{Déroulement du séchage dans les unités}

La préparation des produits destinés au séchage se fait en quatre étapes : le lavage, l'épluchage ou pelage, le découpage et l'étalage sur les claies. La durée de préparation varie fortement d'une unité de séchage à une autre. Pour la préparation de $40 \mathrm{~kg}$ d'ananas (Ananas comusus), elle peut aller de $2 \mathrm{~h}$ 
pour les unes à $3 \mathrm{~h}$ pour d'autres. Cette disparité s'explique par le nombre d'opérateurs alloué à cette tâche et les instruments utilisés pour la préparation des produits.

Le séchage batch, qui consiste à introduire en début de séchage tout le produit à sécher dans la cuve, est le mode de séchage qui se révèle être effectué par toutes les unités enquêtées. Parmi elles, $50 \%$ suivent l'évolution de la température de séchage à l'aide d'un thermomètre analogique. Le séchage des fruits tels que l'ananas (Ananas comusus), la papaye (Carica papaya) et la banane (Musa spp.) se fait en deux étapes dans des séchoirs de modèle Atesta (capacité de $40 \mathrm{~kg}$, Etablissement Taless, Yaoundé, Cameroun) : la première étape dure environ de 4 h à 6 h à une température de séchage voisine de $80^{\circ} \mathrm{C}$; la seconde étape commence ensuite et la température de séchage est alors comprise entre $45^{\circ} \mathrm{C}$ et $55^{\circ} \mathrm{C}$ jusqu'à la fin du séchage. Un cycle de séchage de fruits comme l'ananas peut durer au-delà de 24 h. Les $3 / 4$ des opérateurs essayent de débuter le séchage au plus tard à $14 \mathrm{~h}$ de façon à contrôler ainsi la première étape de séchage avant la tombée de la nuit. Pendant la nuit, la température du séchoir est fixée à $(35 \pm 5){ }^{\circ} \mathrm{C}$ qui est une température proche de la température ambiante. Dès la reprise du travail le lendemain, la température de séchage est à nouveau réglée à $(45 \pm 5){ }^{\circ} \mathrm{C}$ et cela jusqu'à la fin du séchage (figure 5).

Pour réduire le temps de séchage et homogénéiser la qualité du produit séché, les claies chargées de produits sont permu-

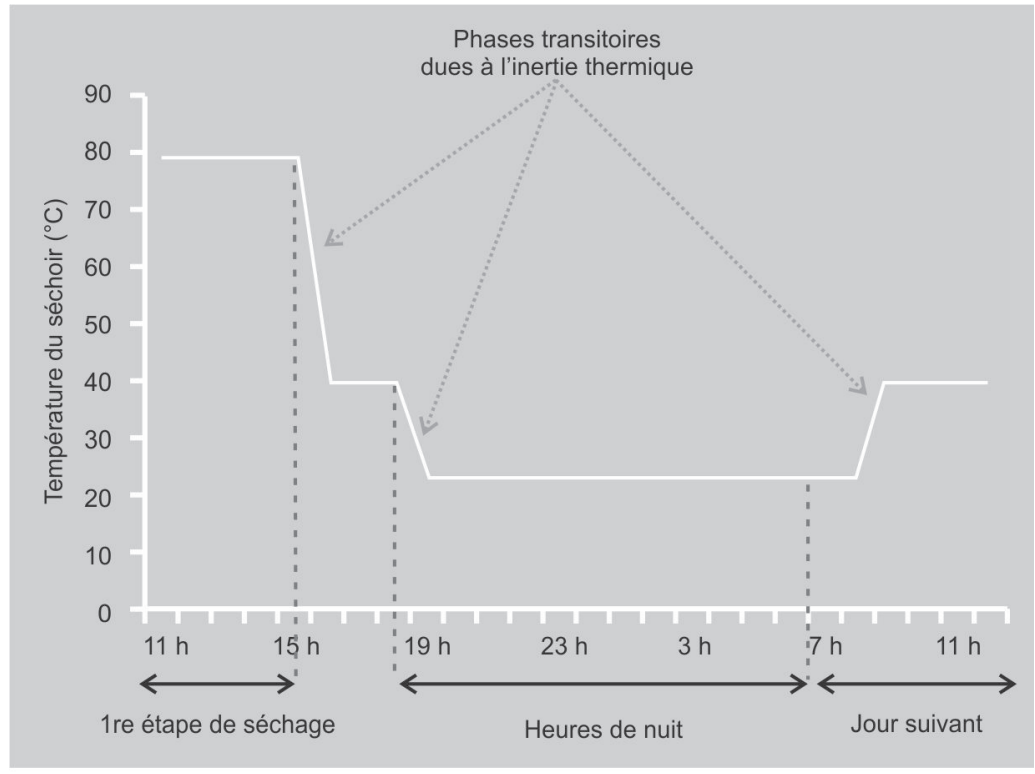

tées toutes les heures, la claie la plus basse venant prendre la place de la plus haute et chacune des autres étant descendue d'un cran tout au long d'un cycle de séchage sauf, bien sûr, durant les heures de nuit.

D'après notre enquête, tous les opérateurs se servent du toucher pour évaluer la fin du séchage en vérifiant si le produit est craquant ou non. De plus, selon leur expérience dans le domaine, ils se servent de la couleur du produit et de son odeur. Cette pratique ne garantit pas une teneur en eau homogène des produits en fin de séchage (tableau III). Pour l'ananas, les teneurs en eau en fin de séchage sont encore

\section{Figure 5.}

Évolution de la température de séchage de $30 \mathrm{~kg}$ d'ananas dans un séchoir de type Atesta (Taless, Yaoundé, Cameroun) dans la ville de Yaoundé (Cameroun), présenté pour un cycle complet de séchage chez un transformateur disposant d'un séchoir à gaz non ventilé [durée du cycle $(24 \pm 2) \mathrm{h}$ ].

\section{Tableau III.}

Comparaison de la teneur en eau du gingembre et de l'ananas séchés, mesurée après un cycle de séchage à l'aide d'un séchoir artisanal dans le sud du Cameroun.

\begin{tabular}{lcc}
\hline Produit & Unité & $\begin{array}{c}\text { Teneur en eau finale } \\
\text { (\% base humide) }\end{array}$ \\
\hline Gingembre séché & GIC Aprodecam & 3 \\
& GIC FADE & 10 \\
Taless Dry Food & 15 \\
Ananas séché & GIC Aprodecam & 29,4 \\
& GIC FADE & 24 \\
& Taless Dry Food & 20
\end{tabular}




\section{Edoun et al.}

\section{Tableau IV.}

Nature, période de forte activité et température de séchage des fruits et légumes les plus soumis au séchage dans le Sud du Cameroun.

\begin{tabular}{|c|c|c|c|c|}
\hline Nature du produit & & $\begin{array}{c}\text { Nombre d'unités } \\
\text { sur } 42 \text { unités étudiées } \\
\text { qui transforment le produit }\end{array}$ & Période de forte activité & $\begin{array}{c}\text { Température } \\
\text { de séchage } \\
\left({ }^{\circ} \mathrm{C}\right)\end{array}$ \\
\hline \multirow[t]{6}{*}{ Fruits } & Ananas & 33 & Mars - novembre & $80-55$ \\
\hline & Mangue & 33 & Février - septembre & \\
\hline & Papaye & 28 & Mai - décembre & \\
\hline & Banane & 28 & Toute l'année & \\
\hline & Safou & 14 & Juillet - décembre & \\
\hline & Noix de coco & 18 & Septembre - février & \\
\hline \multirow[t]{4}{*}{ Légumes } & Carotte & 19 & Décembre - mars & $50-45$ \\
\hline & Gingembre & 13 & Novembre - février & \\
\hline & Piment & 13 & Août - janvier & \\
\hline & Gombo & 9 & Juillet - novembre & \\
\hline \multirow[t]{4}{*}{ Légumes-feuilles } & Ndolè & 35 & Avril - novembre & $50-45$ \\
\hline & Chou & 27 & & \\
\hline & Feuilles de manioc & 27 & & \\
\hline & Okok & 25 & & \\
\hline
\end{tabular}

supérieures à 17,4\%, teneur en eau d'équilibre pour une température moyenne de séchage de $50^{\circ} \mathrm{C}[11]$.

\subsection{Nature des produits séchés}

Les fruits séchés à petite échelle (jusqu'à $500 \mathrm{~kg}$ de produits séchés par cycle de séchage de $24 \mathrm{~h}$ ) dans le sud du Cameroun se sont révélés être principalement l'ananas (A. comusus), la papaye (C.papaya), la banane (Musa spp.), le safou (Dacryodes edulis) et la noix de coco (Cocos nucifera); les légumes : la carotte (Daucus carota var. sativus), le gingembre (Zingiber officinale), le piment (Capsicum sp.) et le gombo (Hibiscus esculentus); les légumes feuilles: le ndolè (Vernonia), le chou (Brassica oleracea), l'okôk (Gnetum spp.) et les feuilles de manioc (Manibot escuelenta) (tableau IV) ; les feuilles-condiments (basilic, persil et céleri) sont séchées en très faible quantité.

Nos observations ont mis en évidence que le séchage à petite échelle (artisanal) des produits constitue encore aujourd'hui un problème mal résolu dès lors qu'il s'agit de produits à haute teneur en eau de type fruits et légumes et que l'on se trouve en zone tropicale où l'humidité de l'air est supérieure à $70 \%$ pendant toutes les périodes de récolte, donc des périodes de forte activité de séchage. Le vecteur du séchage artisanal étant l'air, un simple séchage des produits agricoles à forte teneur en eau, entiers ou fragmentés, montre rapidement ses limites : longue durée du séchage de plusieurs jours, dégradations biochimique des produits, développement des micro-organismes pathogènes favorisé, etc.

\subsection{Les problèmes et besoins des transformateurs}

Les questions à réponses multiples du questionnaire utilisé pour nos enquêtes ont permis de mettre en évidence les divers problèmes rencontrés au quotidien dans les unités de séchage (tableau V). Le problème de pénurie et/ou de rupture de stock de gaz cité par 25 unités sur 42 peut s'expliquer par deux faits majeurs : celui de la pénurie de gaz et celui de la mauvaise organisation du système de fonctionnement des unités de séchage. La crise énergétique en matière de 


\begin{tabular}{|c|c|}
\hline Problème déclaré par les unités de séchage & $\begin{array}{c}\text { Nombre d'unités, sur } 42 \text { unités étudiées, } \\
\text { ayant ce problème }\end{array}$ \\
\hline $\begin{array}{l}\text { Absence de système de régulation des séchoirs } \\
\text { (température et vitesse de l'air de séchage) }\end{array}$ & 38 \\
\hline Pénurie de gaz ou rupture de stock de bouteilles de gaz & 25 \\
\hline Durée de séchage trop longue (supérieure à 24 h) & 25 \\
\hline $\begin{array}{l}\text { Pertes de chaleur à travers les parois } \\
\text { (technologie de fabrication du séchoir) }\end{array}$ & 25 \\
\hline Nécessité d'une surveillance permanente du procédé & 22 \\
\hline Pénibilité de la permutation des claies & 22 \\
\hline $\begin{array}{l}\text { Qualité variable du produit en fin de séchage } \\
\text { (changement de couleur, odeur) }\end{array}$ & 17 \\
\hline Fragilité des claies qui se déchirent pendant le séchage & 12 \\
\hline
\end{tabular}

gaz, que traverse actuellement le Cameroun, se manifeste par des pénuries fréquentes et parfois prolongées. Ce déficit est très sensible dans les grandes métropoles (Douala, Yaoundé, Bafoussam, etc.). Avec une production très marginale (environ $24000 \mathrm{t}$ de gaz par an) et une demande locale de plus en plus forte, le marché est confronté à un réel problème de distribution. Cela contraint parfois les transformateurs à parcourir de longue distance (près de $20 \mathrm{~km}$ ) pour acquérir le combustible. Le système de fonctionnement des unités amplifie cette situation : les unités de séchage à petite échelle au sud du Cameroun ne possèdent généralement qu'une seule bouteille de gaz. Leur approvisionnement en gaz est fait soit $1 j$ avant, soit le jour même du séchage du produit, ce qui ne leur laisse par trop de temps pour trouver une bouteille de gaz dans les environs immédiats de l'unité de séchage.

Les séchoirs installés fonctionnant sans électricité et à convection naturelle, la régulation de la température de l'air se fait par variation du débit de gaz dans le cas d'un séchoir à gaz ou du débit d'air et de biomasse dans le cas d'un séchoir à combustion de biomasse. Dans ce cas, l'inertie thermique du séchoir ne facilite pas la tâche aux opérateurs et les transferts de chaleur airproduit sont difficiles à maîtriser. Le séchage en flux traversant étant le mode de séchage mis en œuvre dans ce type de séchoir, les opérateurs sont contraints de permuter les claies durant le séchage pour homogénéiser la qualité (couleur et teneur en eau) des fruits séchés. La permutation des claies se fait toutes les heures et prend environ 10 min occasionnant des pertes thermiques dues à l'ouverture du battant du séchoir. Il est donc nécessaire qu'un opérateur soit présent durant tout le cycle de séchage.

"La fragilité des claies qui se déchirent pendant le séchage " met en évidence un défaut de conception et une mauvaise qualité des matériaux utilisés pour leur fabrication. Le bois est utilisé pour la fabrication du cadre et de la ficelle en plastique pour la toile. Mais, sous l'effet du poids des produits $\left(8 \mathrm{~kg} \cdot \mathrm{m}^{-2}\right)$ et de la température de séchage élevée $\left(80^{\circ} \mathrm{C}\right)$, les ficelles se dilatent et se cassent.

Le changement de couleur qui donne aux produits un aspect brulé pourrait être expliqué par des réactions de Maillard (réactions non-enzymatiques) et de brunissement enzymatique imputables au fait que 


\section{Edoun et al.}

Tableau VI.

Besoins en séchoir exprimés par des transformateurs de fruits et légumes ne possédant pas de séchoir, selon une enquête réalisée dans le sud du Cameroun.

\begin{tabular}{|c|c|c|c|}
\hline \multirow{2}{*}{$\begin{array}{l}\text { Type de séchoir souhaité } \\
\text { par le transformateur }\end{array}$} & \multicolumn{2}{|c|}{ Caractéristiques des besoins } & \multirow{2}{*}{$\begin{array}{l}\text { Proportion de transformateurs } \\
\text { concernés } \\
(\%)\end{array}$} \\
\hline & $\begin{array}{l}\text { Capacité du séchoir souhaité } \\
\qquad(\mathrm{kg})\end{array}$ & $\begin{array}{l}\text { Temps de séchage souhaité } \\
\text { (h) }\end{array}$ & \\
\hline À gaz ventilé et régulé & 80 à 100 & $\begin{array}{l}\text { Pour les fruits : } 10 \\
\text { Pour les légumes-feuilles : } 1\end{array}$ & 60 \\
\hline À gaz non ventilé & 50 & Pour les fruits : 14 & 10 \\
\hline $\begin{array}{l}\text { À biomasse } \\
\text { (bois ou charbon) }\end{array}$ & 50 à 100 & Pour les fruits : 12 à 14 & 20 \\
\hline $\begin{array}{l}\text { À biomasse } \\
\text { (bois ou charbon) }\end{array}$ & 50 & Non précisée & 10 \\
\hline
\end{tabular}

certains transformateurs sèchent leurs produits à $80{ }^{\circ} \mathrm{C}$ durant tout le cycle de séchage, cette température étant favorable à de telles réactions. Ces observations seraient à rapprocher de celles de Rattanathanalerk et al. sur ananas, selon lesquels une augmentation de la température dans la plage de $55^{\circ} \mathrm{C}$ à $95^{\circ} \mathrm{C}$ et du temps appliqués lors du traitement du produit provoquerait un brunissement du jus [12]. En effet, selon Damasceno Leandro et al., des réactions se déroulant, durant le traitement thermique des fruits, entre les sucres et les acides aminés entraîneraient une réduction de la concentration des sucres, une modification de l'apparence, de la flaveur et une perte de la valeur nutritionnelle [13].

L'analyse des besoins des transformatrices ne possédant pas de séchoir révèle que près de $60 \%$ d'entre elles opteraient pour un séchoir à gaz ventilé d'une capacité supérieure à $50 \mathrm{~kg}$ de produit frais pour un cycle de séchage de 10 h (tableau VI).

Les besoins exprimés par les unités possédant au moins un séchoir sont orientés vers une amélioration de leur installation par réduction du temps de séchage, augmentation de leur capacité de séchage et ajout d'un système de régulation des paramètres de séchage (tableau VII); cela devrait aboutir à une augmentation de la capacité de production de l'unité, un travail facilité, pour des femmes dans la majorité des cas, et, surtout, une amélioration de la qualité des produits obtenus en fin de séchage.

\section{Conclusion}

Nos travaux ont permis de faire un état des lieux des activités de séchage à petite échelle des fruits et légumes dans le sud du Cameroun. Il est apparu que, malgré les différentes offres disponibles localement, le besoin en dispositifs de séchage répondant mieux aux attentes des utilisateurs reste très important. Ce besoin se traduit par une volonté d'acquérir des séchoirs présentant la capacité de traiter plus de $80 \mathrm{~kg}$ de produits frais durant un cycle de séchage de 10 h et d'utiliser comme énergie primaire soit du gaz soit de la biomasse ; il s'ajoute à cela un souhait d'acquérir un système de ventilation et de régulation des paramètres de séchage (demande exprimée par $60 \%$ des transformateurs). Il en ressort que, lors de la conception des modèles présents actuellement sur le marché, le contexte d'usage des séchoirs n'avait pas été pris en compte à partir de tout le processus de transformation des produits.

Pour éviter à l'avenir de possibles rejets de la part des utilisateurs, il sera judicieux de développer et de proposer, aux acteurs de cette activité artisanale, un séchoir original qui prenne en compte l'environnement d'usage du futur équipement tant sur le 


\section{Tableau VII.}

Besoins exprimés par des transformateurs de fruits et légumes possédant au moins un séchoir, selon une enquête réalisée dans le sud du Cameroun.

\begin{tabular}{|c|c|c|c|}
\hline \multirow{2}{*}{$\begin{array}{l}\text { Besoins exprimés } \\
\text { par les transformateurs }\end{array}$} & \multicolumn{2}{|c|}{ Caractéristiques des besoins } & \multirow{2}{*}{$\begin{array}{l}\text { Proportion de transformateurs } \\
\text { concernés } \\
(\%)\end{array}$} \\
\hline & $\begin{array}{l}\text { Capacité du séchoir souhaité } \\
\qquad(\mathrm{kg})\end{array}$ & $\begin{array}{l}\text { Temps de séchage souhaité } \\
\text { (h) }\end{array}$ & \\
\hline $\begin{array}{l}\text { Disposer d'un système de } \\
\text { régulation des paramètres de } \\
\text { séchage }\end{array}$ & - & - & 45 \\
\hline Acquérir un séchoir à gaz ventilé & 80 à 100 & $\begin{array}{l}\text { Pour les fruits : } 10 \\
\text { Pour les légumes : } 1\end{array}$ & 15 \\
\hline $\begin{array}{l}\text { Acquérir un séchoir à gaz non } \\
\text { ventilé }\end{array}$ & 80 & Non précisée & 8 \\
\hline $\begin{array}{l}\text { Acquérir un séchoir à biomasse } \\
\text { (bois ou charbon) }\end{array}$ & 50 à 100 & Pour les fruits : 12 à 14 & 8 \\
\hline Acquérir un séchoir fumoir & 50 à 100 & Temps de fumage : 48 & 24 \\
\hline
\end{tabular}

plan économique, climatique et énergétique que socioculturel. Un cahier des charges fonctionnel issu de l'analyse des usages se révèle indispensable pour véritablement aider la filière des fruits et légumes séchés à se développer. Les degrés de liberté des concepteurs restent importants dans la mesure où il ne semble pas y avoir une pression trop forte sur le coût économique du séchoir à proposer.

\section{Références}

[1] Kuate J., Bella-Manga, Damesse F., Kouodiekong L., Ndindeng S.A., David O., Parrot L., Enquête sur les cultures fruitières dans les exploitations familiales agricoles en zone humide du Cameroun, Fruits 61 (2006) 373-387.

[2] Anon., Document de stratégie de développement du secteur rural, Doc. Princ., Minist. Agric., Yaoundé, Cameroun, 2002, 114 p.

[3] Silou T., Besoins et offre de technologies post-récolte dans l'agroalimentaire en Afrique subsaharienne : Rôle des technologues dans le développement de la petite entreprise, in : 2e Atelier Int. Voies alimentaires d'amélioration des situations nutritionnelles, Presses Univ. Ouagadougou, Burkina Faso, 2003.

[4] Kapseu C., La situation du séchage et des technologies post-récolte en Afrique subsa- harienne, in: Sémin. Rég. Le séchage et technologies post-récolte, Presses Univ. Yaoundé, Ngaoundéré, Cameroun, 2002.

[5] Krokida M.K., Maroulis R.B, Saravacos G.D., The effect of the method of drying on the colour of dehydrated products, Int. J. Food Sci. Technol. 36 (2001) 53-59.

[6] Ekechukwu O.V., Norton B., Review of solarenergy drying systems. II: an overview of solar drying technology, Energ. Convers. Manag. 40 (1999) 615-655.

[7] Bena B., Fuler R.J., Natural convective solar dryer with biomass backup heater, Sol. Energ. 72 (2002) 75-83.

[8] Méot J.M., Marouzé C., Rivier M., Csec-T : un séchoir pour les produits granulés. Principes et méthode de dimensionnement, in : Sémin. Rég. Transformation, conservation et qualité des aliments : Nouvelle approche de lutte contre la pauvreté, GP3A-UCAD-ESP, Dakar, Sénégal, 2007.

[9] Patil R.T., Shukla B.D., A novel design of crop dryer for use in developing countries, Dry. Technol. 24 (5) (2006) 663-669.

[10] Nguyen Duy Lam, Post-harvest research and development in Vietnam, Annu. Rep. Vietnam. Agric., Vietnam Inst. Agric. Eng. Postharvest Technol., Hanoi, Vietnam, 2001.

[11] Talla A., Jannot Y., Elambo-Nkeng G., Puiggali J.-R., Experimental determination and modeling of sorption isotherms of tropical fruits: 


\section{Edoun et al.}

banana, mango and pineapple, Dry. Technol. 23 (8) (2005) 1477-1498.

[12] Rattanathanalerk M., Naphaporn C., Walaiporn S., Effect of thermal processing on the quality loss of pineapple juice, J. Food Eng. 66 (2005) 259-265.
[13] Damasceno Leandro F., Fernandes Fabiano A.N., Magalhães Margarida M.A., Brito Edy S., Non-enzymatic browning in clarified cashew apple juice during thermal treatment: Kinetics and process control, Food Chem. 106 (2007) 172-179.

\section{Práctica del secado artesanal de frutas y verduras en el sur de Camerún.}

Resumen - Introducción. Desde hace algunos años, el secado de frutas y verduras a pequeña escala vuelve a experimentar un interés en el Camerún, tanto para la venta local como para la de la exportación. Sin embargo los transformadores que practican dicha actividad no están satisfechos con los secadores que emplean. Material y métodos. En este contexto, realizamos una pesquisa de tipo semi abierto, basada en aproximadamente cincuenta unidades de secado, instaladas en las zonas de Duala y de Yaundé (Camerún), con el fin de identificar mejor las dificultades con las que se topan. Se codificaron las informaciones obtenidas en el curso de dicha pesquisa para facilitar su explotación informática. En el muestreo constituido solamente conservamos las empresas que trabajaban a pequeña escala (el 70\% del muestreo inicial), es decir las que trataban menos de $500 \mathrm{~kg}$ de productos frescos por ciclo de secado. Resultados. El análisis de los resultados mostró que cerca del 45\% de las unidades estudiadas no poseían secador alguno y que el $70 \%$ de los secadores recopilados en las unidades eran secadores de gas. En conjunto, cerca del $92 \%$ de estos secadores son secadores de convección natural. El modelo de secador conocido es el modelo Atesta, importado de Burkina Faso, del cual se fabrican variantes a nivel local. Resulta que dicho modelo sólo responde parcialmente a las necesidades de los usuarios y que los transformadores locales abandonen cada vez más estos secadores. Conclusión. El reconocimiento de las actividades de secado a pequeña escala de las frutas y verduras en el sur de Camerún ha hecho que resalte el hecho de que siga siendo muy importante la necesidad de dispositivos de secado, que ofrezcan una mejor respuesta a las expectativas de los usuarios, a pesar de las diferentes ofertas disponibles localmente. Dicha necesidad se traduce por una voluntad de adquirir secadores que presenten la capacidad de tratar más de $80 \mathrm{~kg}$ de productos frescos durante un ciclo de secado de $10 \mathrm{~h}$, y que estén dotados de un sistema de ventilación y de regulación de parámetros de secado.

Camerún / trópicos húmedos / frutas tropicales / hortalizas / secado deshidratación / secado por ventilación / secadoras / artesanía / empresas peque ̃as 